\title{
A RECORDING AND ANALYSIS SYSTEM OF BIOPTIC DRIVING BEHAVIORS
}

\author{
Gang Luo ${ }^{1}$, Xianping $\mathrm{Fu}^{1,2}$ \& Eli Peli ${ }^{1}$ \\ ${ }^{1}$ Schepens Eye Research Institute \\ Department of Ophthalmology, Harvard Medical School \\ Boston, Massachusetts, USA \\ ${ }^{2}$ Information Science and Technology College, \\ Dalian Maritime University, Dalian, China \\ Email: gang.luo@schepens.harvard.edu
}

\begin{abstract}
Summary: Millions of visually impaired people do not drive because they fail to meet the general vision requirements. There is a legal option in 38 US states where people with moderate central vision loss (e.g. visual acuity better than 20/200) may be permitted to drive while wearing spectacle-mounted bioptic telescopes. However, the safety of bioptic driving is still highly controversial, because bioptic use in driving is not well understood. Whether and how bioptic telescopes are actually used in driving, how they should be used appropriately, and whether their use results in better or worse driving performance has never been scientifically established. We are developing an in-car camera system that can be installed in bioptic drivers' own vehicles to record their daily driving activities over long periods of time. Videos of the driver and traffic, GPS coordinates, XYZ acceleration, and vehicle black box data are recorded. We are also developing computer-aided reviewing techniques to automatically identify the most informative driving segments from the vast amount of data and, reconstruct the selected driving maneuvers on an interactive interface, so that these representative segments can be assessed off-line by driver evaluation and training specialists.
\end{abstract}

\section{INTRODUCTION}

Based on the demographics from the 2000 US Census, an estimated 2.4 millions American adults have low vision (visual acuity worse than $20 / 40$ in the better eye), typically caused by conditions such as age-related macular degeneration, optic atrophy and diabetic retinopathy. (Congdon et al., 2004). Because of failure to meet the general vision requirements for licensing, many people lose their driving privileges. However, in 38 US states people with moderate vision loss may be allowed to drive with bioptic telescopes (Peli \& Peli, 2002). The spectacle-mounted bioptic telescopes were introduced in the early 1970s as a driving aid (Kelleher, Mehr, \& Hirsch, 1971). The magnification provided by the telescope compensates for reduced visual acuity (Figure 1). Most of the time, the users view and scan the environment through the regular spectacle lenses, with an unrestricted field of vision. They look intermittently through the telescope (achieved by a slight downward tilt of the head for 1 to $2 \mathrm{sec}$.; Figure $1 \mathrm{~b}$ ) to read road signs, determine the status of traffic lights, or scan ahead for road hazards.

The safety of driving with bioptic telescopes is still highly controversial. Advocates believe proper use can provide users with the necessary sight for driving (Bailey, 1985; Huss \& Corn, 
2004; Kelleher et al., 1971), while opponents strongly suspect that the use of telescope cause failures to see traffic, and that bioptic drivers use telescopes purely as a means for licensing but
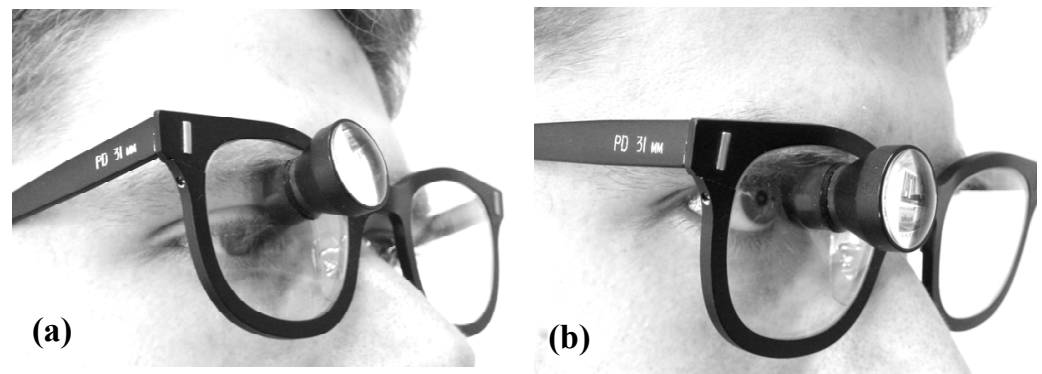

Figure 1. A bioptic telescope used for driving. (a)The driver views below the telescope most of the time. (b) Looking only intermittently and briefly through the telescope by a downward tilt of the head.

not for driving (Fonda, 1995; Tallman, 1984). Retrospective studies that have compared accident rates of bioptic drivers and matched control drivers resulted in inconsistent conclusions - some found bioptic drivers had fewer or no more accidents than the general public (Janke, 1983; Korb, 1970) while the others found they were involved in more accidents (Barron, 1991; Lippmann, Corn, \& Lewis, 1988). These studies do not provide any information about how or why the accidents happened. For example, it still needs to be answered whether the accidents of bioptic drivers occurred when looking through the telescope tube (which might cause a failure to see traffic in periphery), or occurred because of failures to look through the telescope (which might result in poor perception of important traffic situations).

Since bioptic telescopes are designed to be used infrequently, conventional on-board driving assessments may be inappropriate or too limited. Existing knowledge and training guidelines are based on self-reports from bioptic drivers and opinions from experts, but there is insufficient evidence to support any bioptic usage pattern as optimal. We believe that comprehensive observations of actual use of bioptic telescopes based on daily driving are necessary. In a preliminary study, we used a VCR-based in-car camera system to observe how bioptic telescopes were used by two bioptic drivers in their daily driving activities (Luo \& Peli, 2008), and compared our observations with their reports in a survey (Bowers, Apfelbaum, \& Peli, 2005). As for how much driving time they spent while actually looking through the telescope, they reported 60 times and 3 times more than actual use, respectively. We also observed that the use of a cell phone during driving reduced the use of the telescope. These findings highlight the importance of recording of actual bioptic driving behaviors.

We are currently developing a digital in-car camera system that features a large recording capacity, higher quality videos, and recording of a variety of information (including GPS, vehicle black box, and XYZ acceleration). The system will be installed in bioptic drivers' own vehicles to record their actual daily driving activities across long periods of time (such as two months). The driving procedures will be reconstructed using the multi-channel data for off-line assessment. Long-term recording of daily driving activities will result in thousands of hours of videos for multiple subjects. It would be impractical to manually review every second of those videos. Thus we are also developing computer-aided video reviewing techniques to greatly reduce the work load and tedium of reviewing the lengthy clips. 


\section{METHODS}

\section{In-car camera system}

The in-car camera system can be easily installed in subjects' own cars and uninstalled afterwards (Figure 2). It is based on a mobile digital video recorder. In addition to recording 4-channel videos separately in MPEG4 format, it can record GPS locations at $0.5 \mathrm{~Hz}, \mathrm{XYZ}$ acceleration at $30 \mathrm{~Hz}$, vehicle speed, and multiple ON/OFF signals (including turn signals and braking) from the car. Two side-by-side cameras mounted in front of the rear-view mirror combine to provide an $80^{\circ} \times 32^{\circ}$ view forward of traffic and road conditions. They are used to better match the horizontally wide and vertically restricted view the driver has through the windshield (about $90^{\circ} \times 30^{\circ}$ (Vargas-Martin \& Garcia-Perez, 2005). If a single camera with a 4:3 aspect ratio were used to capture the wide scene, a large portion of the images would be empty sky, and the resolution of road images would be low. Thus, it would be difficult for us to interpret what objects are viewed through the telescopes. There are two additional cameras mounted at the two sides of the windshield, aimed at the driver's head. Images from the two angles allow us to track the driver's head movement.
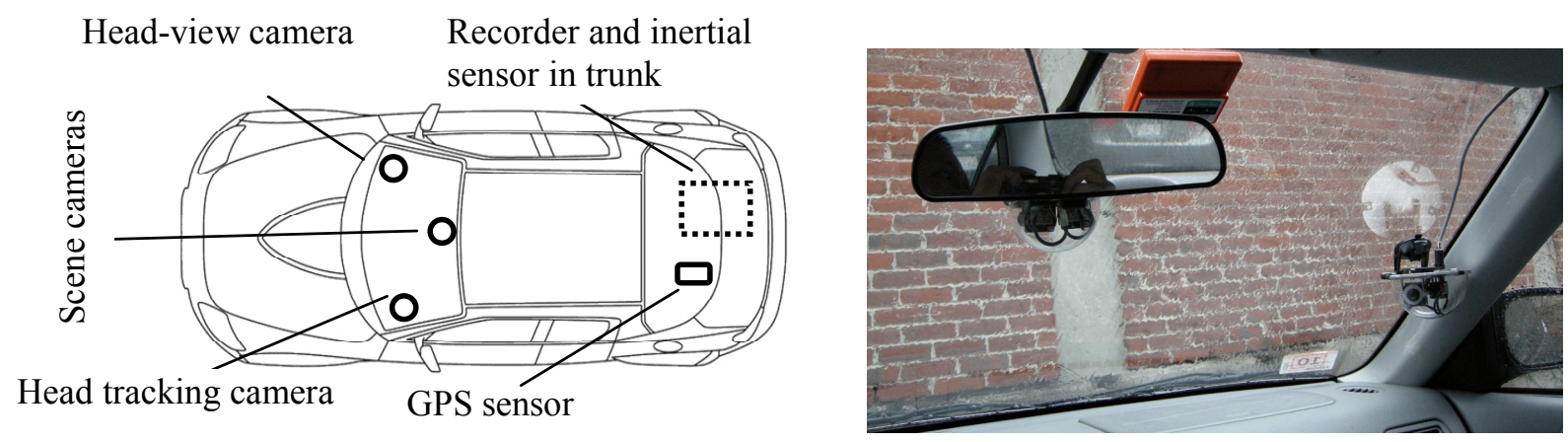

Figure 2. The digital in-car camera system is comprised of two cameras for the road and two for the driver's head mounted on the windshield, and a DVR in the trunk of the car. The system can also record GPS, XYZ acceleration, and vehicle black box data.

\section{Reviewing interface}

To provide as much information as possible in the most effective manner for off-line reviewing, we have been developing an interactive reviewing interface. As illustrated in Figure 3, videos from the side by side scene cameras provide a wide view of the road. Speed and status of turn signals and brake are shown. Acceleration is also plotted. Vehicle location and route information is dynamically marked on a local map to provide orientation. Reviewers can pause or rewind the videos, and vehicle status and location is synchronized with the videos.

\section{Head tracking}

Looking through the telescope is expected to be an infrequent event (e.g. 1\% of driving time), yet of the utmost interest for bioptic driving studies. Knowing when the events occur and what objects are examined through telescopes may be critical for understanding bioptic driving. We have developed a series of image processing techniques to track head movement based on 
fiducial markers pasted on the spectacle frame. The markers ensure high robustness of the image processing. Our head tracking techniques allow free translational head movement (moving back/forth, side to side). As illustrated in Figure 4, we are able to not only detect telescope use by detecting quick downward head movements, but also estimate gaze points through the telescope. In driving studies, bioptic use detected by automatic processing will be manually verified by checking if the eye pupil is aligned with the telescope tube based on head video images. The estimated gaze points will be shown on the scene view and manually interpreted based on scene content. For example, if there is a road sign near the estimated gaze point, it is a reasonable interpretation that the driver is looking at the sign.

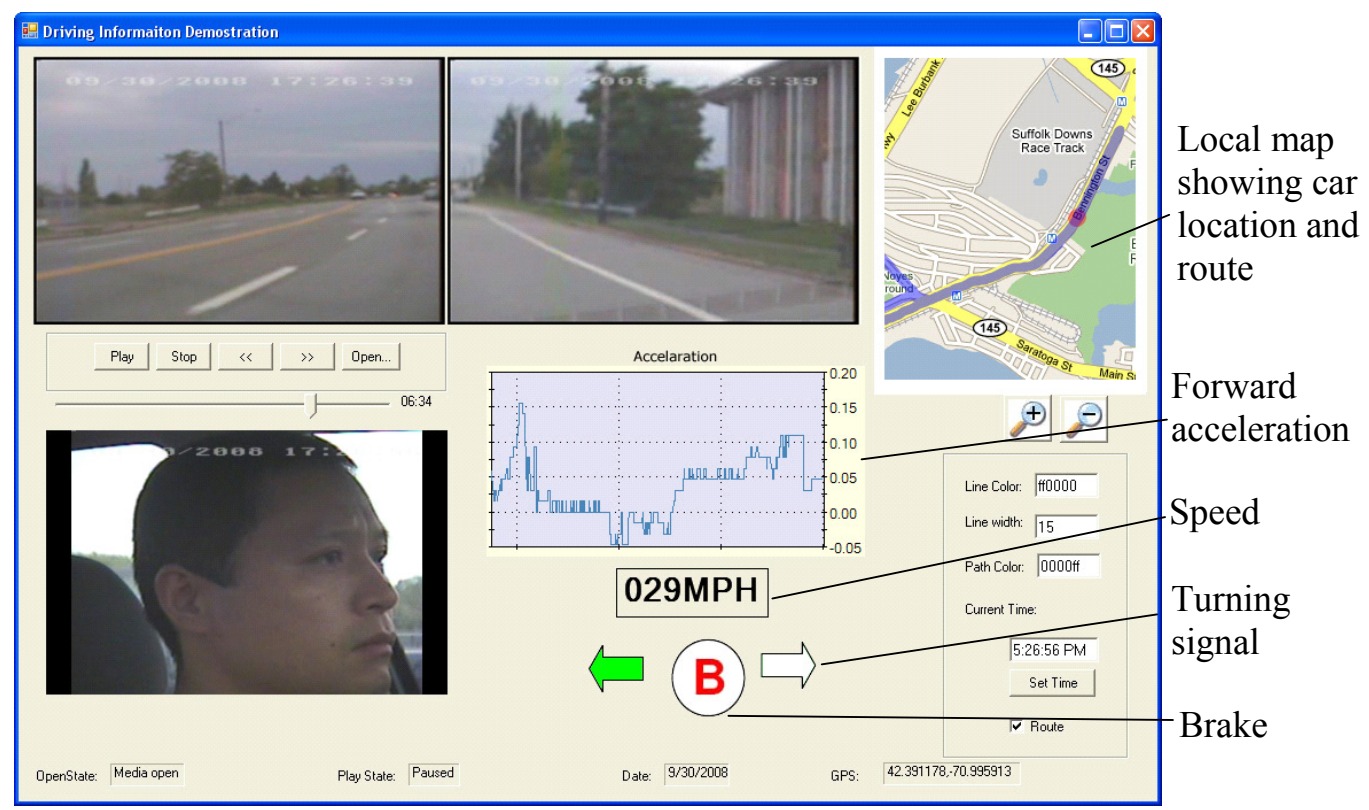

Figure 3. Reviewing interface that presents videos of the road scene and the driver's head, car location and driving route on street map, speed, acceleration, status of turn signals and brake. Users can pause or rewind videos, and all other information will be synchronized with the video.

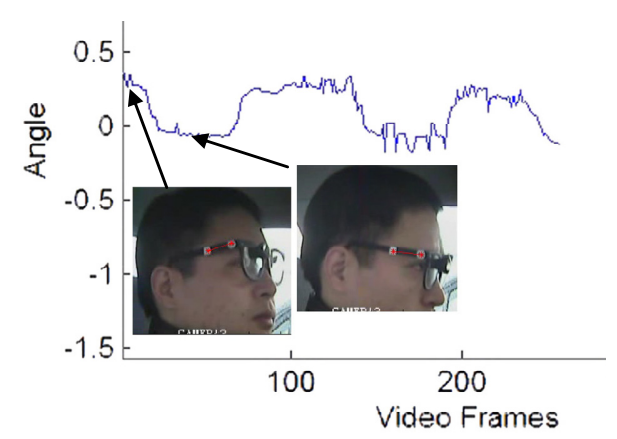

(a)
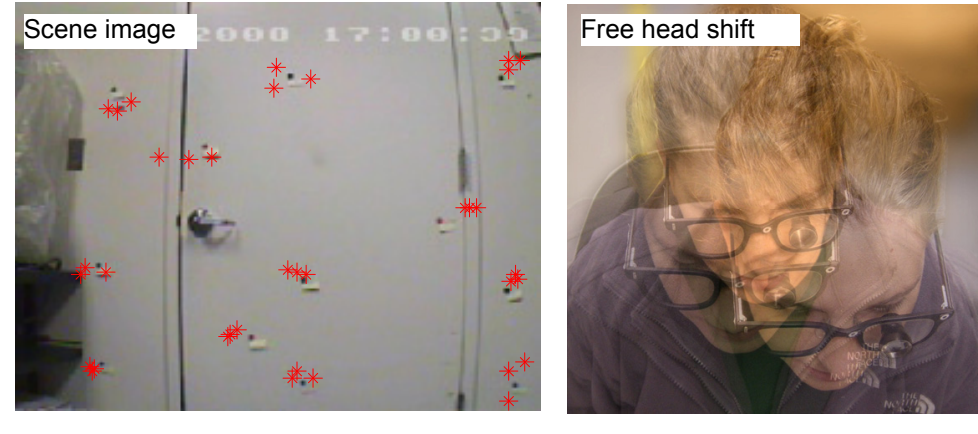

(b)

Figure 4. Head tracking techniques based on fiducial markers attached on the spectacle frame. (a) Bioptic use is noted by a quick downwards head tilt. (b) An example of estimation of gaze points through telescope in an indoor experiment. Three different head positions were recorded for each black dot target on the scene image, and the estimated gaze points (asterisks) were all close to the targets. The field of view of the scene was about $67^{\circ}$ wide. Note that our technique is not affected by the distortion of the scene camera. 


\section{Automated segment identification and selection}

Manual reviewing of unedited videos would take a much longer time than video playback because careful analyses are needed. Such manual analyses would be impractical. Therefore, it is necessary to carefully review only the most informative segments. In the 100-car study (Neale, Dingus, Klauer, \& Goodman, 2005 ), drivers were asked to push an incident button so that reviewers were able to directly access those incident video segments. We propose to automatically and objectively identify informative segments based on the multi-channel data recorded by our system. Here we present some of the techniques we have developed for automated segment selection.

Driving through intersections is important for assessment of driving skills (Al-Ghamdi, 2003; Hakamies-Blomqvist, 1993). Skipping many cruising segments and closely watching intersection/interchange segments will greatly reduce the assessment workload. We have developed a technique that can identify these segments. A critical element to support this technique is a database of geographic coordinates of all of the 654,491 intersections and interchanges in the New England area (Massachusetts, Vermont, New Hampshire, Maine, and Rhode Island). The database also includes road class information (e.g. highway, major road, local street), which will allow us to select intersections by road class for different analyses (Figure 5). Since our recorded GPS data are saved along with a time tag, the corresponding video segments can be extracted.
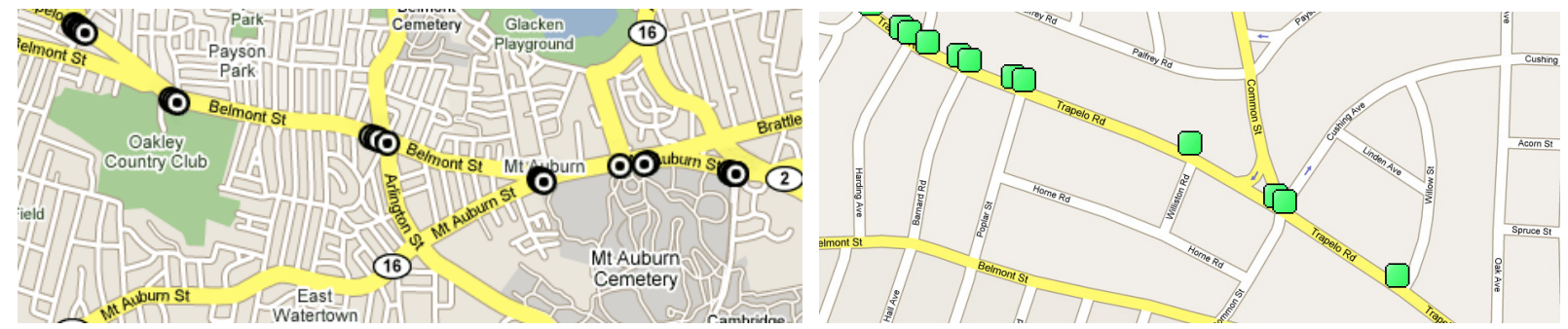

Figure 5. A technique has been developed to identify from GPS recordings segments when the vehicle crosses intersections. The class of roads can be specified, for example, to select only major road intersections (circle markers on the left figure), or to also include minor street intersections (green markers on the right figure).

Maintaining lane position is an important driving skill. On the other hand, steering changes may also occur in response to certain traffic situations (e.g. avoiding a dog running across the road), which require awareness of surrounding situations, correct judgment, sufficient vision and appropriate operation. Therefore, steering change segments are informative for assessment of driving skills. These segments can be automatically identified based on lateral inertial fluctuation. For instance, the weaving maneuver is characterized by a sinusoid pattern in the lateral inertial signal (Figure 6). 

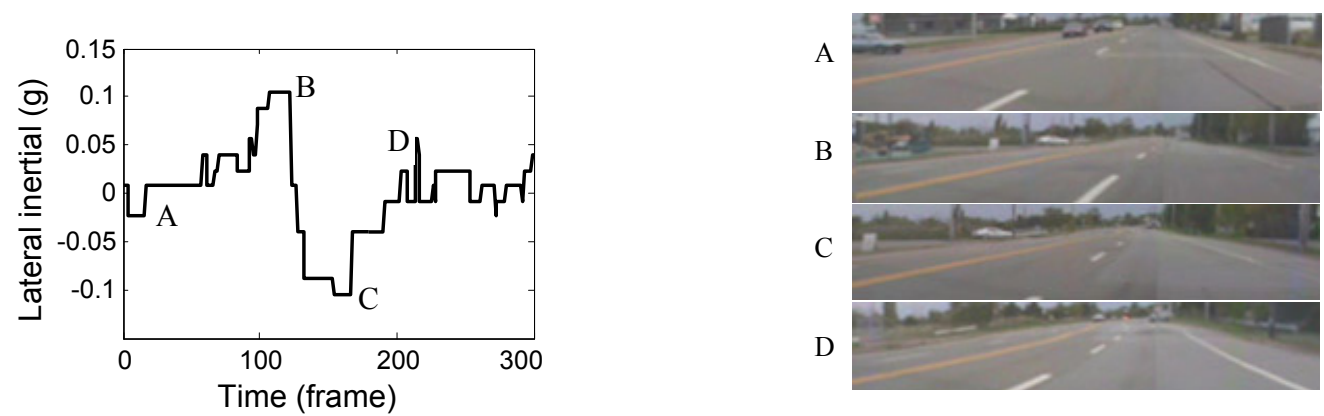

Figure 6. Detection of weaving based on lateral inertial fluctuation. This figures show an example where the car veered to the left and then repositioned. This event is featured by a sinusoid pattern in the lateral acceleration signal. Note the shifting horizontal position of the lane marker at the bottom of the video images.

\section{RESULTS}

Figure 7 shows a driving trip that was automatically annotated by the segment identification process. Turning, braking, bioptic use, weaving, etc are labeled along the driving distance. Some of the results are directly plotted using recorded data, e.g. brake and turn signals, and the others are analysis results based on recorded information, e.g. turning maneuver, weaving and rapid stop.

To address specific questions, different segments may need to be selected in driving studies. We can easily use different search criteria to select targeted segments for different purposes based on the segment annotation results. As shown in Figure 7a, for instance, three types of segments within the $5200 \mathrm{~m}$ driving trip can be identified using criteria combining turn signals, turning maneuver and intersection information. Here we show turning without signaling (TNS), signaled lane changing (CL), and driving through major intersections without turning (TI). The satellite picture of a major intersection on the trip is shown in Figure 7b, superimposed with the trip route and TNS-, CL-, TI-segments occurred at the location.

\section{DISCUSSIONS}

The recording and analysis system is still under development. When completed it should reveal never-before-seen bioptic use behaviors. Our long term goal is to assess the value of bioptic telescope for driving safety. Accidents are very rare incidents. For example, the high profile 100car study only recorded 82 crashes and 761 near-crashes with 43,000 hours of recording from 241 drivers over one year (Neale et al., 2005 ). We believe that off-line assessment of general driving performance based on naturalistic driving can reliably indicate drivers' skills, and predict safety. With much less data than the 100-car study, many questions regarding bioptic driving safety could be addressed. We are working with driving instructors to develop the off-line assessment system, which will be validated against commonly used on-board assessments. In the iterative tuning procedure of the off-line assessment system, more data collection and analyses will be required. 

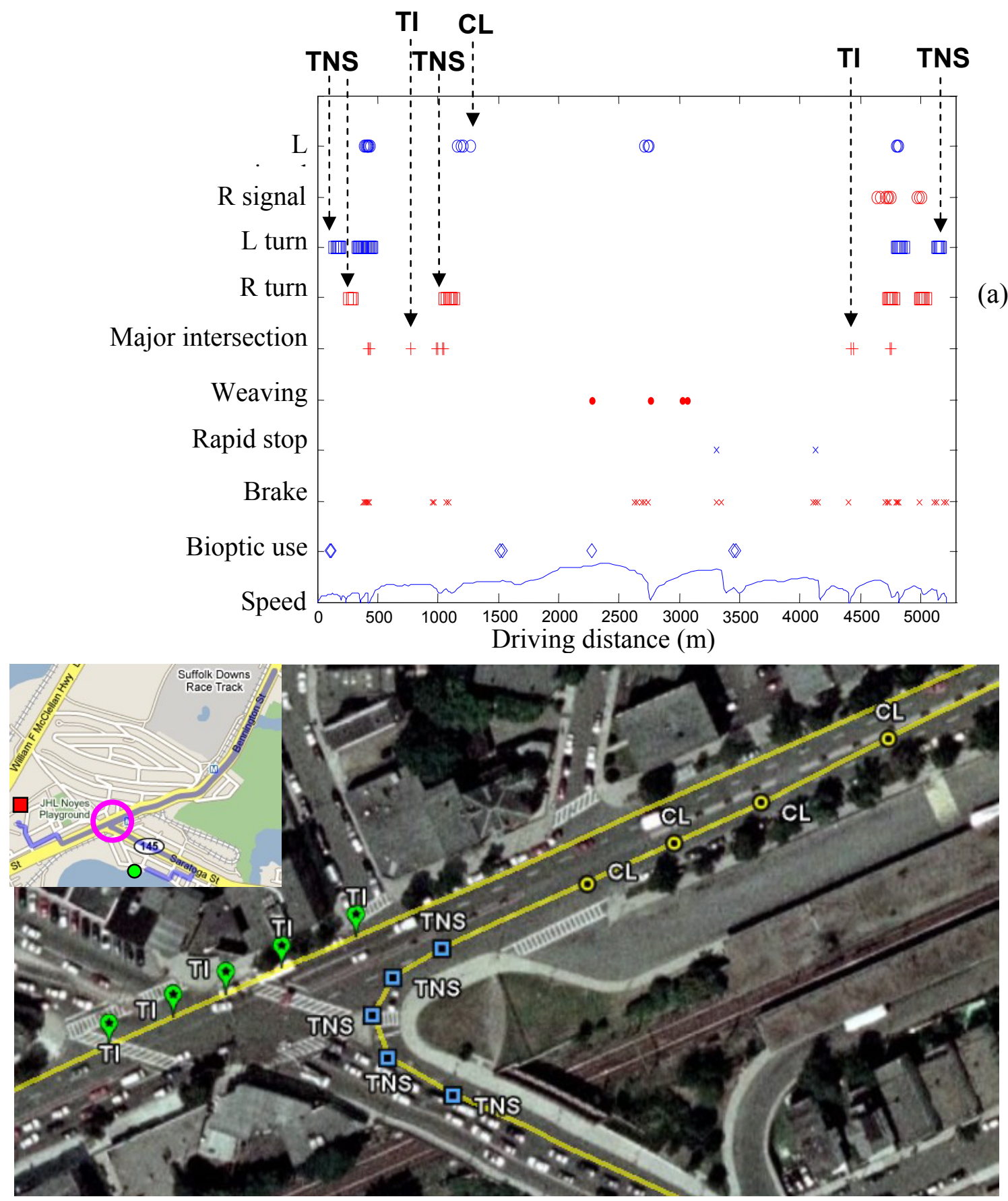

(b)

Figure 7. Driving segment identification and selection. (a) An example of recorded data and analysis results of a 5200m driving trip. Using Boolean operation, specific segments can be identified for different purposes, e.g. turning without signaling (TNS), signaled lane changing (CL), and driving through major intersections (TI). (b) The satellite picture of a major intersection on the trip. TNS, CL and TI segments all occurred at the location. The inset map indicates the driving route and the location of the intersection.

\section{ACKNOWLEDGMENT}

Supported by NIH grants EY12890 and Mass Lions foundation. 


\section{REFERENCES:}

Al-Ghamdi, A. S. (2003). Analysis of traffic accidents at urban intersections in Riyadh. Accid Anal Prev, 35(5), 717-724.

Bailey, I. (1985). Bioptic telescopes. Arch. Ophthalmol., 103(1), 13-14.

Barron, C. (1991). Bioptic telescopic spectacles for motor vehicle driving. J Am Optom Assoc, $62(1), 37-41$.

Bowers, A. R., Apfelbaum, D. H., \& Peli, E. (2005). Bioptic telescopes meet the needs of drivers with moderate visual acuity loss. Investigative Ophthalmology \& Visual Science, 46(1), 6674.

Congdon, N., O'Colmain, B., Klaver, C. C. W., Klein, R., Munoz, B., Friedman, D. S., et al. (2004). Causes and prevalence of visual impairment among adults in the United States. Archives of Ophthalmology, 122(4), 477-485.

Fonda, G. (1995). Bioptic Telescopes Should Not Be Recommended So That Visually-Impaired Persons Can Drive. Journal of Visual Impairment \& Blindness, 89(6), 485-486.

Hakamies-Blomqvist, L. E. (1993). Fatal accidents of older drivers. Accid Anal Prev, 25(1), 1927.

Huss, C., \& Corn, A. (2004). Low vision driving with bioptics: An overview. Journal of Visual Impairment \& Blindness, 98(10), 641-653.

Janke, M. (1983). Accident rates of drivers with bioptic telescopic lenses. Journal of Safety Research, 14, 159-165.

Kelleher, D. K., Mehr, E. B., \& Hirsch, M. J. (1971). Motor Vehicle Operation by a Patient with Low Vision - Case Report. American Journal of Optometry and Archives of American Academy of Optometry, 48(9), 773-776.

Korb, D. R. (1970). Preparing Visually Handicapped Person for Motor Vehicle Operation. American Journal of Optometry and Archives of American Academy of Optometry, 47(8), 619.

Lippmann, O., Corn, A. L., \& Lewis, M. C. (1988). Bioptic Telescopic Spectacles and Driving Performance - a Study in Texas. Journal of Visual Impairment \& Blindness, 82(5), 182-187.

Luo, G., \& Peli, E. (2008). Application of video surveillance systems to reveal actual use of bioptic telescopes in driving. Paper presented at the Vision 2008.

Neale, V., Dingus, T., Klauer, J., \& Goodman, M. (2005 ). An Overview of The 100-Car Naturalistic Study and Findings. Paper presented at the 19th ESV Conference.

Peli, E., \& Peli, D. (2002). Driving with confidence: a practical guide to driving with low vision: Singapore: World Scientific Publishing Company.

Tallman, C. B. (1984). Bioptic telescopic spectacle: a hazard for operating a motor vehicle. Arch Ophthalmol, 102(8), 1119-1120.

Vargas-Martin, F., \& Garcia-Perez, M. A. (2005). Visual fields at the wheel. Optometry and Vision Science, 82(8), 675-681. 